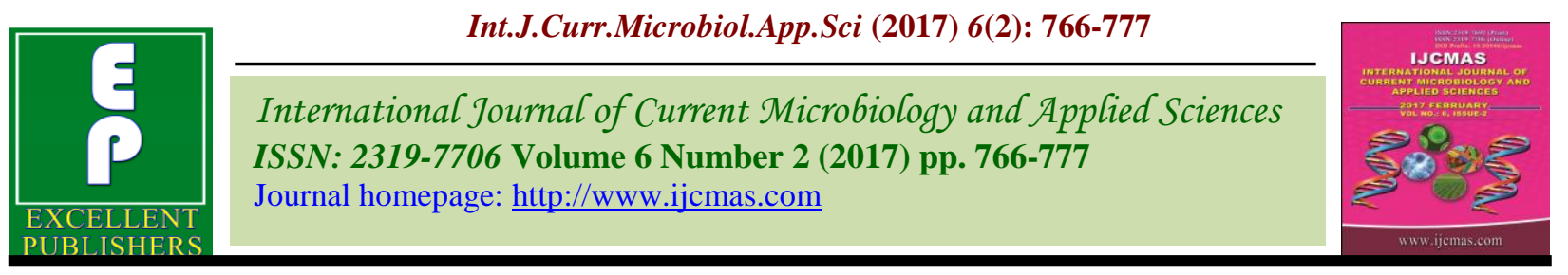

Original Research Article

http://dx.doi.org/10.20546/ijcmas.2017.602.085

\title{
Advance Agro- Techniques for Yield Efficiency and Economics of Potato (Solanum tuberosum) Crop for Chhattisgarh Plains
}

\author{
C.K. Chandrakar*, G.K. Shrivastava, S.K. Dwivedi, D. Sharma, \\ K.K. Pandey and S.K. Singh \\ Indira Gandhi Krishi Vishwavidyalaya, Raipur (C.G.), India \\ *Corresponding author
}

\section{A B S T R A C T}

\section{Keywords \\ Growth parameters, Herbicide, Irrigation, Organic manure etc.}

\section{Article Info}

Accepted:

18 January 2017

Available Online:

10 February 2017
The experiment has been conducted on the experimental field of Indira Gandhi Krishi Vishwavidyalaya, Raipur under split-split plot design along with three replications in the year 2010-11 and 2011-12 during the Rabi season in potato crops (Solanum tuberosum). Irrigation (three treatments) as a main plot, weed management (four treatments) as a sub plot and Integrated Nutrient Management (INM) (four treatments) has been taken as subsub plot in the spacing of $60 \times 20 \mathrm{~cm}$ on the variety Chipsona- 2 . Total combined plots were $3 \times 4 \times 4 \times 3=144$. The parameters have been taken: growth parameter i.e. CGR, tuberization efficiency, weed parameters: weed index, weed control efficiency, nutrient uptake, yield and yield Attributes i.e. No. of Stolen/plant, no. of tubers/plants, tuber yield/ha and economics. The result revealed that the significantly higher CGR, tuberization efficiency, nutrient uptake by plants, yield has been found under the $75 \% \mathrm{~N}$ (inorganic) $+25 \% \mathrm{~N}$ (poultry manure) + PSB + Azotobactor in sub-sub plot, the herbicide metribuzin found most efficient and effective than the other herbicide i.e. under sub plot. Drip irrigation has been found significant than other method of Irrigation; on the different parameter of growth, yield and yield attributes as well. The recommendation of the study is potato crop performed better under drip irrigation 100 and $125 \%$ of OPE, $75 \% \mathrm{~N}$ (inorganic) $+25 \% \mathrm{~N}$ (poultry manure) + PSB + Azotobactor with application of metribuzin $\left(500 \mathrm{~g}\right.$ a.i ha $\left.{ }^{-1}\right)$ herbicide with the spacing of $60 \times 20 \mathrm{~cm}$ for the plains of Chhattisgarh.

\section{Introduction}

Potato (Solanum tuberosum) is the most important food and vegetable cum starch supplying crop of the world. It is one of the most remunerative and profitable crop for the growers due to its higher yield potential within a limited time. India has $2^{\text {nd }}$ rank in production $3^{\text {rd }}$ in area and $4^{\text {th }}$ in productivity in the world with export of 15424 lacks (IIVR-2015). Water is the vital source for crop production and is the most limiting factor in Indian agricultural scenario. Though
India has the largest irrigation network, the irrigation efficiency has not been achieved more than 40 per cent. Due to water scarcity, the available water resources should be very effectively utilized through water saving irrigation technologies. Hence, further expansion of irrigation may depend upon the adoption of new systems such as pressurized irrigation methods with the limited water resources. Amongst those pressurized irrigation methods, drip irrigation has proved 
its superiority over other methods of irrigation due to the direct application of water and nutrients in the vicinity of root zone. There are several constraints in potato production, of which weeds often pose a serious problem. Weeds not only compete with crop plants for nutrients, soil moisture, space and sunlight but also serve as an alternative hosts for several insect pest and diseases. Hand weeding and hoeing are common practices followed in India. However, timely weed control may not be possible manually due to non-availability of labours and high rate of wages during peak period of farm operations. Hence, chemical weed control appears to hold a great promise in dealing with effective, timely and economic weed suppression. Similar work has been done by Chandrakar et al., (2012) and reported that same result on the potato crop for Chhattisgarh. The overall strategy for increasing potato yields and sustaining them at a high level must include an integrated approach to the management of soil nutrients, along with other complementary measures.

\section{Materials and Methods}

The field experiment was conducted at Indira Gandhi Krishi Vishwavidyalaya, Raipur (C.G) during rabi 2010-11 and 2011-12. The soil of experimental site was clay loam in texture, neutral in soil reaction, low in available $\mathrm{N}$, and low in available $\mathrm{P}$ and high in available $\mathrm{K}$ status. The climate of the region is sub humid with an average annual rainfall of 1200-1400 $\mathrm{mm}$. The crop received approximately $65.0 \mathrm{~mm}$ rainfall during crop period.

The experiment on Kufri Chipsona- 2 with the spacing $60 \mathrm{~cm} \times 20 \mathrm{~cm}$ and experimental layout has given below:

\begin{tabular}{|c|c|c|c|}
\hline \multirow{3}{*}{ Main plot } & \multirow{3}{*}{ Irrigation } & Drip irrigation ( $125 \%$ of OPE) & $\mathrm{I}_{1}$ \\
\hline & & Drip irrigation (100\% of OPE) & $\mathrm{I}_{2}$ \\
\hline & & Control (furrow irrigation) & $\mathrm{I}_{3}$ \\
\hline \multirow{4}{*}{ Sub plot } & \multirow{4}{*}{$\begin{array}{l}\text { Weed } \\
\text { management }\end{array}$} & Weedy check & $\mathrm{W}_{0}$ \\
\hline & & Hand weeding (at 25 and 45 DAP) & $\mathrm{W}_{1}$ \\
\hline & & Metribuzin $\left(500 \mathrm{~g}\right.$ a.i. ha $\left.^{-1} \mathrm{PE}\right)$ & $\mathrm{W}_{2}$ \\
\hline & & Chlorimuron + quizalofop $\left(6+50 \mathrm{~g}\right.$ a.i ha $\left.{ }^{-1}\right)$ & $\mathrm{W}_{3}$ \\
\hline \multirow{4}{*}{$\begin{array}{l}\text { Sub- Sub } \\
\text { plot }\end{array}$} & \multirow{4}{*}{$\begin{array}{l}\text { Integrated } \\
\text { Nutrient } \\
\text { Management }\end{array}$} & $100 \% \mathrm{RDF}$ & $\mathrm{F}_{1}$ \\
\hline & & $\begin{array}{l}100 \% \text { RDF + Micro nutrient (Zinc sulphate } 25 \mathrm{~kg} \mathrm{ha}^{-} \\
{ }_{1} \text { ) }\end{array}$ & $\mathrm{F}_{2}$ \\
\hline & & $\begin{array}{l}75 \% \mathrm{~N} \text { inorganic fertilizer }+25 \% \mathrm{~N} \text { poultry manure } \\
+\mathrm{PSB}+\text { Azatobactor }\end{array}$ & $\mathrm{F}_{3}$ \\
\hline & & $\begin{array}{l}50 \% \mathrm{~N} \text { inorganic fertilizer }+50 \% \mathrm{~N} \text { poultry manure } \\
+\mathrm{PSB}+\text { Azatobactor }\end{array}$ & $\mathrm{F}_{4}$ \\
\hline
\end{tabular}

The observations of different growth parameters and yield parameters in both the years were recorded. A sample consisting of five plants was selected at random from each net plot. The observations on plant height, number of shoots plant ${ }^{-1}$ and number of leaves plant $^{-1}$ were recorded on these randomly selected plants from each plot of each replication separately (at 20,40,60 DAP and at maturity). Per cent emergence was noted by counting the emerged plant at 20 DAP. The observations on fresh weight of shoots plant ${ }^{-1}$, fresh weight of tubers plant ${ }^{-1}$, fresh weight of roots plant ${ }^{-1}$, dry weight of shoots plants ${ }^{-1}$, dry weight of roots plant ${ }^{-1}$, number of stolons plant $^{-1}$, LAI, CGR, number of tubers plant ${ }^{-1}$, 
fresh and dry weight of tuber plant ${ }^{-1}$ were recorded from three randomly selected and uprooted plants in each plot in each replication separately. Marketable, unmarketable and total tuber yield were recorded on net plot basis in each replication separately.

Crop growth rate $\left(\mathrm{g} \mathrm{day}^{-1}\right.$ plant $\left.^{-1}\right)$ is the rate of dry matter production per unit ground area per unit time and was computed by following formula:

$\underset{\left(\text { g day }^{-1} \text { plant }^{-1}\right)}{\text { Crop growth rate }(C G R)}=\frac{\mathrm{W}_{2}-\mathrm{W}_{1}}{\mathrm{t}_{2}-\mathrm{t}_{1}}$

Where, $\mathrm{W}_{1}$ and $\mathrm{W}_{2}$ are dry weight of plants at time $t_{1}$ and $t_{2}$, respectively.

Tuberization efficiency was calculated by dividing tuber weight with shoot weight

Tuberization efficiency $=$

Fresh weight of tubers plant ${ }^{-1}(\mathrm{~g})$

Fresh weight of shoots plant ${ }^{-1}(\mathrm{~g})$

\section{Results and Discussion}

\section{Crop growth rate and tuberization efficiency}

The finding revealed that crop growth rate and tuberization efficiency (Tuber: Haulm ratio) were significantly influenced due to irrigation schedule, weed and integrated nutrient management (Table 1). The crop growth rate and tuberization efficiency were significantly superior under drip irrigation (125\% of Open pan evaporation) over furrow irrigation but it was at par with drip irrigation (100\% of Open pan evaporation) during both the years and in mean data. This is due to supply of moisture was found sufficient throughout the crop growth stages for the treatment which received by drip irrigation High solar radiation interception enhanced the plant for high photosynthesis and causes the higher dry matter accumulation in tubers. This result reaffirmed the findings of many earlier workers such as El Saidi et al., (2010)

As regards to weed management, Metribuzin (500 g a.i. ha-1PE) showed significantly higher crop growth rate as well as tuberization efficiency as compared to weedy check and rest of the treatments during both the years and on mean basis. The main reason behind this was due to significant impact of Metribuzin (500g a.i ha-1 P.E) which also resulted the maximum weed control efficiency. With the application of this treatment, maximum weeds were controlled timely, leading to utilization of maximum resources by potato plants which was reflected through maximum growth parameters by this treatment. Similar findings were also reported by Ahmed et al., (2011).

Application of $75 \% \mathrm{~N}$ inorganic fertilizer + $25 \% \mathrm{~N}$ organic (Poultry manure) + PSB + Azotobactor produced significantly higher crop growth rate and tuberization efficiency than other nutrient management practices during both the years as well as in mean analysis. This may be due to an increased availability of nutrients to the plant in the presence of biofertilizers and organic manure (poultry manure) These findings are in agreement with those reported earlier by Sarkar et al., (2011).

\section{Effect on weed parameters}

\section{Weed species}

The field was dominated with broad leaf weeds; the major weed species existed in the experimental area was Chenopodium album, Convolvulus arvensis, Melilotus alba, Medicago denticulata, Cynodon dactylon, and 
others etc., (Table 2). Out of five weed species Cynodon dactylon was in grass group and rests were broad leaf weeds.

\section{Weed index and weed control efficiency}

The data depicted in table 3 indicate that weed index (WI) was significantly higher when crop was imposed with furrow irrigation as compared to drip irrigation (100 $\%$ of OPE) and drip irrigation (125\% of OPE), however both the drip irrigation treatment recorded statistically similar weed index during both the years and also in mean analysis. Among weed management practices, weed index (WI) was observed significantly lowest under Metribuzin (500g a.i ha ${ }^{-1}$ P.E. followed by two hand weeding (at 25 and 45 DAP). Whereas significantly highest weed index was found under weedy check during both the years and on mean data.

Among integrated nutrient management, least weed index (WI) was found under $75 \% \mathrm{~N}$ inorganic fertilizer $+25 \% \mathrm{~N}$ organic (Poultry manure) + PSB + Azotobactor, whereas, maximum weed index (WI) was noted under $100 \%$ RDF during both the years and in case of mean data.

The data presented in table 3 indicate that weed control efficiency (WCE) was significantly highest when crop was imposed with drip irrigation (100\% Of OPE) followed by drip irrigation (125\% of OPE), however, furrow irrigation showed significantly least weed control efficiency during both the years and on mean basis. Among weed management practices, weed control efficiency (WCE) was observed maximum under Metribuzin $(500 \mathrm{~g}$ a.i ha ${ }^{-1}$ P.E. followed by two hand weeding (at 25 and 45 DAP) during both the years and on mean basis. Integrated nutrient management practices and interaction effect did not show significant effect on weed control efficiency during both the years as well as in mean analysis. Higher weed control efficiency under Metribuzin (500 g a.i. ha-1PE) was due to better control of weeds, which was also reflected through reduced weed dry weight under this treatment. Similar findings were also reported by Singh et al., (2007) and Roder et al., (2009).

\section{Nutrient uptake $(\mathrm{N}, \mathrm{P}, \mathrm{K})\left(\mathrm{kg} \mathrm{ha}^{-1}\right)$ by potato crop}

Nutrient uptake $\left(\mathrm{kg} \mathrm{ha}^{-1}\right)$ by potato crop (plant + tuber) is shown in table 4 Significantly higher nutrient uptake (nitrogen $(\mathrm{N})$, phosphorus $(\mathrm{P})$, potash $(\mathrm{K}))$ by crop was found when the crop was imposed treatment with drip irrigation (125\% of OPE) followed by drip irrigation (100\% of OPE) and minimum was noted in furrow irrigation. As regards to weed management practices, the entire major nutrients (nitrogen, phosphorus and potassium) uptake was found significantly higher under application of Metribuzin (500 g a.i. ha ${ }^{-1} \mathrm{PE}$ ) as compared to weedy check and rest of the treatments during both the years and in mean data. The lowest uptake was recorded in weedy check. Among integrated nutrient management practices, application of $75 \% \mathrm{~N}$ inorganic fertilizer $+25 \% \mathrm{~N}$ organic (Poultry manure) $+\mathrm{PSB}+$ Azotobactor produced significantly higher uptake of all major nutrients (nitrogen, phosphorus and potassium) than other nutrient management practices during both the years and on mean basis. The lowest uptake was obtained by $100 \%$ RDF. The maximum uptake by plant and tuber in above treatment might be due to supplemental addition of $\mathrm{N}$ through atmospheric $\mathrm{N}$ fixation in the soil by Azotobactor, and PSB would have caused more mobilization and solubilization of insoluble $\mathrm{P}$ in the soil and improved the availability of $\mathrm{P}$, which may have resulted in an increased uptake of $\mathrm{P}$ by plants. The $\mathrm{K}$ uptake was also maximum by plant and tuber. Similarly, with the application of $75 \% \mathrm{~N}$ inorganic fertilizer $+25 \% \mathrm{~N}$ organic (Poultry manure) $+\mathrm{PSB}+$ Azotobactor also increased 
the NPK content in soil. The results corroborate the findings of Parmar et al., (2007) and Swalin et al., (2008).

\section{Yield attributes and yield}

Irrigation schedule positively influenced the yield attributes and yield. The number of stolons plant ${ }^{-1}$ (28.35), number of tubers plant 1(14.57) and tuber yield (31.49 t/ha) were significantly higher under drip irrigation (125 $\%$ of open pan evaporation) than control (furrow irrigation) but was at par with drip irrigation (100\% of open pan evaporation) during both the years and on mean basis.

Table.1 Effect of irrigation schedule, weed and integrated nutrient management on Crop Growth Rate CGR (g day-1) and tuberization efficiency

\begin{tabular}{|c|c|c|c|c|c|c|}
\hline \multirow[t]{2}{*}{ Treatment } & \multicolumn{3}{|c|}{ CGR $\left(\right.$ g day $\left.^{-1}\right)$} & \multicolumn{3}{|c|}{$\begin{array}{l}\text { Tuberization Efficiency } \\
\text { (Tuber: Haulm ratio) }\end{array}$} \\
\hline & 2010-11 & 2011-12 & Mean & 2010-11 & 2011-12 & Mean \\
\hline \multicolumn{7}{|l|}{ Irrigation schedule } \\
\hline $\mathrm{I}_{1}-$ & 10.58 & 8.57 & 9.57 & 2.14 & 2.11 & 2.13 \\
\hline $\mathrm{I}_{2}-$ & 11.01 & 8.90 & 9.95 & 2.16 & 2.12 & 2.14 \\
\hline $\mathrm{I}_{3}-$ & 5.90 & 4.61 & 5.26 & 1.80 & 1.82 & 1.81 \\
\hline SEm \pm & 0.11 & 0.22 & 0.16 & 0.03 & 0.03 & 0.03 \\
\hline $\mathrm{CD}(\mathrm{P}=\mathbf{0 . 0 5})$ & 0.44 & 0.85 & 0.63 & 0.14 & 0.15 & 0.14 \\
\hline \multicolumn{7}{|l|}{ Weed management } \\
\hline $\mathrm{W}_{0}-$ & 8.65 & 6.86 & 7.75 & 1.90 & 1.88 & 1.89 \\
\hline $\mathrm{W}_{1}-$ & 9.56 & 7.47 & 8.51 & 2.16 & 2.13 & 2.14 \\
\hline $\mathrm{W}_{2}-$ & 9.71 & 8.03 & 8.87 & 2.17 & 2.17 & 2.16 \\
\hline $\mathrm{W}_{3}-$ & 8.74 & 7.08 & 7.91 & 1.92 & 1.89 & 1.90 \\
\hline $\operatorname{SEm} \pm$ & 0.23 & 0.20 & 0.21 & 0.03 & 0.02 & 0.03 \\
\hline $\mathrm{CD}(\mathrm{P}=0.05)$ & 0.68 & 0.60 & 0.62 & 0.09 & 0.08 & 0.08 \\
\hline \multirow{2}{*}{\multicolumn{7}{|c|}{$\begin{array}{l}\text { Integrated } \\
\text { nutrient management }\end{array}$}} \\
\hline & & & & & & \\
\hline $\mathrm{F}_{2}-$ & 8.96 & 7.25 & 8.10 & 2.03 & 1.99 & 2.01 \\
\hline $\mathrm{F}_{3}-$ & 10.04 & 8.05 & 9.04 & 2.13 & 2.13 & 2.13 \\
\hline $\mathrm{F}_{4}-$ & 9.11 & 7.30 & 8.21 & 2.02 & 2.00 & 2.01 \\
\hline SEm \pm & 0.28 & 0.26 & 0.26 & 0.03 & 0.03 & 0.03 \\
\hline $\mathrm{CD}(\mathrm{P}=0.05)$ & 0.80 & 0.73 & 0.73 & 0.09 & 0.09 & 0.08 \\
\hline
\end{tabular}


Table.2 List of weed flora reported in experimental field

\begin{tabular}{|l|l|l|l|l|}
\hline S. No. & \multicolumn{1}{|c|}{ Group } & \multicolumn{1}{|c|}{ Botanical Name } & \multicolumn{1}{|c|}{ Family } & \multicolumn{1}{c|}{$\begin{array}{c}\text { Vernacular } \\
\text { Name }\end{array}$} \\
\cline { 1 - 3 } 1 & \multirow{2}{*}{ Broad leaf weeds } & Chenopodium album & Fabaceae & Bathua \\
\cline { 4 - 5 } & & Convolvulus arvensis & Convolvulaceae & Hirankhuri \\
\cline { 4 - 5 } & & Melilotus alba & Fabaceae & Safed senji \\
\cline { 4 - 5 } 3 & & Medicago denticulate & Fabaceae & Chinori \\
\hline 5 & & Cynodon dactylon & Poaceae & Doob \\
\hline 5 & & &
\end{tabular}

Table.3 Effect of Irrigation schedule, weed and integrated nutrient management on weed control efficiency and weed index

\begin{tabular}{|c|c|c|c|c|c|c|}
\hline \multirow[t]{2}{*}{ Treatment } & \multicolumn{3}{|c|}{$\begin{array}{c}\text { Weed control efficiency } \\
(\%)\end{array}$} & \multicolumn{3}{|c|}{ Weed index } \\
\hline & 2010-11 & 2011-12 & Mean & 2010-11 & 2011-12 & Mean \\
\hline Irrigation schedule & & & & & & \\
\hline & 77.9 & 81.4 & 79.6 & 5.80 & 5.72 & 5.76 \\
\hline $\mathrm{I}_{2}-$ & 74.7 & 78.7 & 76.7 & 4.94 & 4.95 & 4.94 \\
\hline $\mathrm{I}_{3}-$ & 50.0 & 51.0 & 50.6 & 15.22 & 15.28 & 15.25 \\
\hline SEm \pm & 0.67 & 1.82 & 0.64 & 0.23 & 0.24 & 0.23 \\
\hline $\mathrm{CD}(\mathrm{P}=\mathbf{0 . 0 5})$ & 2.62 & 7.11 & 2.50 & 0.90 & 0.94 & 0.92 \\
\hline Weed management & & & & & & \\
\hline $\mathrm{W}_{0}-$ & -- & -- & -- & 11.15 & 11.27 & 11.21 \\
\hline $\mathrm{W}_{1}-$ & 78.1 & 79.7 & 78.9 & 7.38 & 7.48 & 7.43 \\
\hline $\mathrm{W}_{2}-$ & 85.4 & 87.8 & 86.6 & 6.44 & 6.36 & 6.40 \\
\hline $\mathrm{W}_{3}-$ & 63.4 & 64.5 & 69.7 & 9.63 & 9.48 & 9.56 \\
\hline SEm \pm & 0.88 & 1.30 & 0.83 & 0.19 & 0.20 & 0.19 \\
\hline $\mathrm{CD}(\mathrm{P}=0.05)$ & 2.61 & 3.25 & 2.48 & 0.57 & 0.59 & 0.58 \\
\hline Integrated & & & & & & \\
\hline $\begin{array}{l}\text { nutrient managemen } \\
\mathrm{F}_{1}-\end{array}$ & 68.1 & 68.9 & 68.5 & 11.11 & 11.14 & 11.12 \\
\hline $\mathrm{F}_{2}-$ & 69.2 & 70.7 & 70.0 & 9.35 & 9.33 & 9.34 \\
\hline $\mathrm{F}_{3}-$ & 69.6 & 71.3 & 70.3 & 5.51 & 5.40 & 5.46 \\
\hline $\mathrm{F}_{4}-$ & 69.0 & 71.4 & 70.8 & 8.65 & 8.73 & 8.69 \\
\hline SEm \pm & 1.00 & 1.03 & 0.82 & 0.27 & 0.26 & 0.26 \\
\hline $\mathrm{CD}(\bar{P}=0.05)$ & NS & $\mathrm{NS}$ & NS & 0.77 & 0.75 & 0.74 \\
\hline
\end{tabular}


Table.4 Effect of irrigation schedule, weed and integrated nutrient management on nutrient uptake by potato crop

\begin{tabular}{|l|c|c|c|c|c|c|c|c|c|}
\hline Treatment & \multicolumn{3}{|c|}{ Nitrogen $(\mathrm{Kg} / \mathrm{ha})$} & \multicolumn{3}{c|}{ Phosphorus(Kg/ha) } & \multicolumn{3}{c|}{ Potash(Kg/ha) } \\
\hline & $\mathbf{2 0 1 0 - 1 1}$ & $\mathbf{2 0 1 1 - 1 2}$ & Mean & $\mathbf{2 0 1 0 - 1 1}$ & $\mathbf{2 0 1 1 - 1 2}$ & Mean & $\mathbf{2 0 1 0 - 1 1}$ & $\mathbf{2 0 1 1 - 1 2}$ & Mean \\
\hline Irrigation schedule & & & & & & & & & \\
$\mathrm{I}_{1}-$ & 210.61 & 223.23 & 216.92 & 52.60 & 57.49 & 55.04 & 283.04 & 301.64 & 292.34 \\
$\mathrm{I}_{2}-$ & 218.63 & 234.54 & 226.58 & 56.19 & 61.16 & 58.67 & 292.52 & 312.86 & 302.69 \\
$\mathrm{I}_{3}-$ & 120.79 & 129.01 & 124.90 & 25.95 & 28.43 & 27.19 & 175.05 & 190.69 & 182.87 \\
SEm \pm & 2.35 & 2.40 & 2.37 & 1.90 & 2.33 & 2.05 & 2.55 & 2.80 & 2.65 \\
CD $(\mathbf{P}=\mathbf{0 . 0 5})$ & 9.17 & 9.37 & 9.26 & 7.44 & 9.11 & 8.03 & 9.97 & 10.95 & 10.36 \\
\hline Weed management & & & & & & & & & \\
$\mathrm{W}_{0}-$ & 151.95 & 160.31 & 156.13 & 32.51 & 36.10 & 34.31 & 202.11 & 211.18 & 206.65 \\
$\mathrm{~W}_{1}-$ & 197.01 & 208.60 & 202.81 & 49.36 & 54.12 & 51.74 & 271.12 & 286.77 & 278.95 \\
$\mathrm{~W}_{2}-$ & 212.22 & 230.55 & 221.39 & 56.66 & 61.54 & 59.10 & 287.92 & 318.09 & 303.01 \\
$\mathrm{~W}_{3}-$ & 172.20 & 182.90 & 177.55 & 41.12 & 44.35 & 42.73 & 239.67 & 257.54 & 248.61 \\
SEm \pm & 3.03 & 3.28 & 3.14 & 1.99 & 2.07 & 1.99 & 3.12 & 3.71 & 3.36 \\
CD $(\mathbf{P}=\mathbf{0 . 0 5})$ & 9.00 & 9.77 & 9.34 & 5.92 & 6.15 & 5.92 & 9.28 & 11.03 & 9.99 \\
\hline Integrated & & & & & & & & & \\
nutrient management & & 165.08 & & & & & & & \\
$F_{1}-$ & 154.14 & 180.06 & 159.61 & 36.50 & 40.29 & 38.40 & 214.60 & 230.75 & 222.68 \\
$F_{2}-$ & 168.36 & 244.72 & 174.21 & 39.94 & 44.60 & 42.26 & 233.10 & 249.30 & 241.20 \\
$\mathrm{~F}_{3}-$ & 230.36 & 192.52 & 237.54 & 56.72 & 61.53 & 59.12 & 306.69 & 327.65 & 317.17 \\
$\mathrm{~F}_{4}-$ & 180.51 & 3.24 & 186.51 & 45.18 & 49.11 & 47.14 & 246.43 & 265.89 & 256.16 \\
SEm \pm & 2.96 & 9.14 & 3.08 & 1.37 & 1.54 & 1.40 & 3.21 & 3.45 & 3.29 \\
CD $(\mathbf{P}=\mathbf{0 . 0 5})$ & 8.34 & & 8.70 & 3.87 & 4.35 & 3.95 & 9.06 & 9.73 & 9.28 \\
\hline
\end{tabular}

Table.5 Effect of Irrigation schedule, weed and integrated nutrient management on yield attributes and tuber yield

\begin{tabular}{|c|c|c|c|c|c|c|c|c|c|}
\hline \multirow[t]{2}{*}{ Treatment } & \multicolumn{3}{|c|}{$\begin{array}{c}\text { Number of stolons } \\
\text { plant }^{-1}\end{array}$} & \multicolumn{3}{|c|}{ Number of tubers plant ${ }^{-1}$} & \multicolumn{3}{|c|}{ Tuber yield $\left(\mathrm{t} \mathrm{ha}^{-1}\right)$} \\
\hline & 2010-11 & 2011-12 & Mean & 2010-11 & 2011-12 & Mean & 2010-11 & 2011-12 & Mean \\
\hline $\begin{array}{l}\text { Irrigation schedule } \\
\mathrm{I}_{1}-\end{array}$ & 26.47 & 29.38 & 27.91 & 12.43 & 15.63 & 14.03 & 30.16 & 31.24 & 30.59 \\
\hline $\mathrm{I}_{2}-$ & 26.98 & 29.72 & 28.35 & 13.00 & 16.13 & 14.57 & 31.02 & 32.01 & 31.49 \\
\hline $\mathrm{I}_{3}-$ & 24.37 & 26.39 & 25.37 & 9.15 & 11.56 & 10.35 & 20.74 & 21.68 & 21.21 \\
\hline SEm \pm & 0.13 & 0.36 & 0.17 & 0.23 & 0.18 & 0.20 & 0.23 & 0.24 & 0.23 \\
\hline $\mathrm{CD}(\mathrm{P}=\mathbf{0 . 0 5})$ & 0.53 & 1.41 & 0.66 & 0.90 & 0.72 & 0.78 & 0.91 & 0.93 & 0.92 \\
\hline Weed management & & & & & & & & & \\
\hline $\mathrm{W}_{0}-$ & 24.44 & 26.19 & 25.30 & 10.14 & 12.90 & 11.52 & 24.81 & 25.68 & 25.25 \\
\hline $\mathrm{W}_{1-}$ & 26.35 & 28.88 & 27.59 & 12.30 & 15.04 & 13.67 & 28.57 & 29.48 & 28.96 \\
\hline $\mathrm{W}_{2}-$ & 26.86 & 30.83 & 28.85 & 12.95 & 16.28 & 14.62 & 29.51 & 30.60 & 29.99 \\
\hline $\mathrm{W}_{3}-$ & 26.11 & 28.10 & 27.10 & 10.71 & 13.54 & 12.13 & 26.33 & 27.47 & 26.87 \\
\hline SEm \pm & 0.13 & 0.24 & 0.16 & 0.12 & 0.14 & 0.13 & 0.19 & 0.20 & 0.20 \\
\hline $\mathrm{CD}(\mathrm{P}=\mathbf{0 . 0 5})$ & 0.39 & 0.74 & 0.49 & 0.37 & 0.43 & 0.39 & 0.57 & 0.59 & 0.59 \\
\hline $\begin{array}{l}\text { Integrated } \\
\text { nutrient management }\end{array}$ & & & & & & & & & \\
\hline $\mathrm{F}_{1}-$ & 24.82 & 27.17 & 25.97 & 10.50 & 13.49 & 12.00 & 24.85 & 25.82 & 25.30 \\
\hline $\mathrm{F}_{2}-$ & 25.51 & 28.11 & 26.81 & 10.87 & 13.82 & 12.35 & 26.61 & 27.63 & 27.08 \\
\hline $\mathrm{F}_{3}-$ & 27.70 & 30.52 & 29.11 & 13.55 & 16.54 & 15.05 & 30.45 & 31.58 & 30.96 \\
\hline $\mathrm{F}_{4}-$ & 25.73 & 28.19 & 26.95 & 11.18 & 13.91 & 12.55 & 27.31 & 28.23 & 27.73 \\
\hline SEm \pm & 0.16 & 0.24 & 0.17 & 0.18 & 0.17 & 0.17 & 0.273 & 0.26 & 0.26 \\
\hline $\mathrm{CD}(\overline{\mathrm{P}}=0.05)$ & 0.45 & 0.69 & 0.49 & 0.52 & 0.49 & 0.48 & 0.769 & 0.75 & $\mathbf{0 . 7 3}$ \\
\hline
\end{tabular}


Table.6 Effect of Irrigation schedule, weed and integrated nutrient management on economics of potato crop

\begin{tabular}{|c|c|c|c|c|c|c|c|c|c|}
\hline Treatment & Cost of & $\begin{array}{l}\text { cultivat } \\
\text { ha }^{-1} \text { ) }\end{array}$ & ion $(\mathrm{Rs}$ & Net in & come $(\mathbf{R}$ & $\left(\mathbf{s h}^{-1}\right)$ & & B:C ratic & \\
\hline & 2010-11 & 2011-12 & Mean & 2010-11 & \begin{tabular}{|l|} 
2011-12 \\
\end{tabular} & Mean & 2010-11 & |2011-12 & Mean \\
\hline $\begin{array}{l}\text { Irrigation schedule } \\
\mathrm{I}_{1}- \\
\mathrm{I}_{2}- \\
\mathrm{I}_{3}-\end{array}$ & $\begin{array}{l}67775 \\
69114 \\
59199\end{array}$ & $\begin{array}{l}68535 \\
69874 \\
61039\end{array}$ & $\begin{array}{l}68130 \\
69494 \\
60119\end{array}$ & $\begin{array}{c}107095 \\
110769 \\
59671\end{array}$ & $\begin{array}{c}127580 \\
131084 \\
73640\end{array}$ & $\begin{array}{c}117338 \\
120926 \\
66655\end{array}$ & $\begin{array}{l}1.58 \\
1.60 \\
1.00\end{array}$ & $\begin{array}{l}1.86 \\
1.87 \\
1.20\end{array}$ & $\begin{array}{l}1.72 \\
1.73 \\
1.10\end{array}$ \\
\hline $\begin{array}{l}\text { Weed management } \\
\mathrm{W}_{0-} \\
\mathrm{W}_{1}- \\
\mathrm{W}_{2}- \\
\mathrm{W}_{3}-\end{array}$ & $\begin{array}{l}63936 \\
68106 \\
64964 \\
64444\end{array}$ & $\begin{array}{l}65039 \\
69209 \\
66117 \\
65567\end{array}$ & $\begin{array}{l}64487 \\
68657 \\
65540 \\
65005\end{array}$ & $\begin{array}{c}79352 \\
97238 \\
105541 \\
89916\end{array}$ & $\begin{array}{c}95971 \\
115459 \\
125393 \\
106249\end{array}$ & $\begin{array}{c}87662 \\
106349 \\
115467 \\
97082\end{array}$ & $\begin{array}{l}1.21 \\
1.41 \\
1.60 \\
1.34\end{array}$ & $\begin{array}{l}1.45 \\
1.66 \\
1.88 \\
1.60\end{array}$ & $\begin{array}{l}1.33 \\
1.53 \\
1.74 \\
1.47\end{array}$ \\
\hline $\begin{array}{l}\text { Integrated } \\
\text { nutrient managemei } \\
\mathrm{F}_{1}- \\
\mathrm{F}_{2}- \\
\mathrm{F}_{3}- \\
\mathrm{F}_{4}-\end{array}$ & $\begin{array}{l}64953 \\
65753 \\
65399 \\
65345\end{array}$ & $\begin{array}{l}66063 \\
66913 \\
66506 \\
66449\end{array}$ & $\begin{array}{l}65508 \\
66333 \\
65953 \\
65897 \\
\end{array}$ & $\begin{array}{c}78669 \\
88224 \\
110672 \\
92482 \\
\end{array}$ & $\begin{array}{c}95653 \\
106059 \\
131177 \\
110183 \\
\end{array}$ & $\begin{array}{c}87161 \\
97142 \\
120925 \\
101332 \\
\end{array}$ & $\begin{array}{l}1.18 \\
1.31 \\
1.68 \\
1.39 \\
\end{array}$ & $\begin{array}{l}1.42 \\
1.56 \\
1.96 \\
1.63 \\
\end{array}$ & $\begin{array}{l}1.30 \\
1.44 \\
1.82 \\
1.51 \\
\end{array}$ \\
\hline
\end{tabular}

Fig.1 Effect of interaction between irrigation schedule (I) and weed management

(W) on total tuber yield of potato ( $\mathrm{t}$ ha-1)

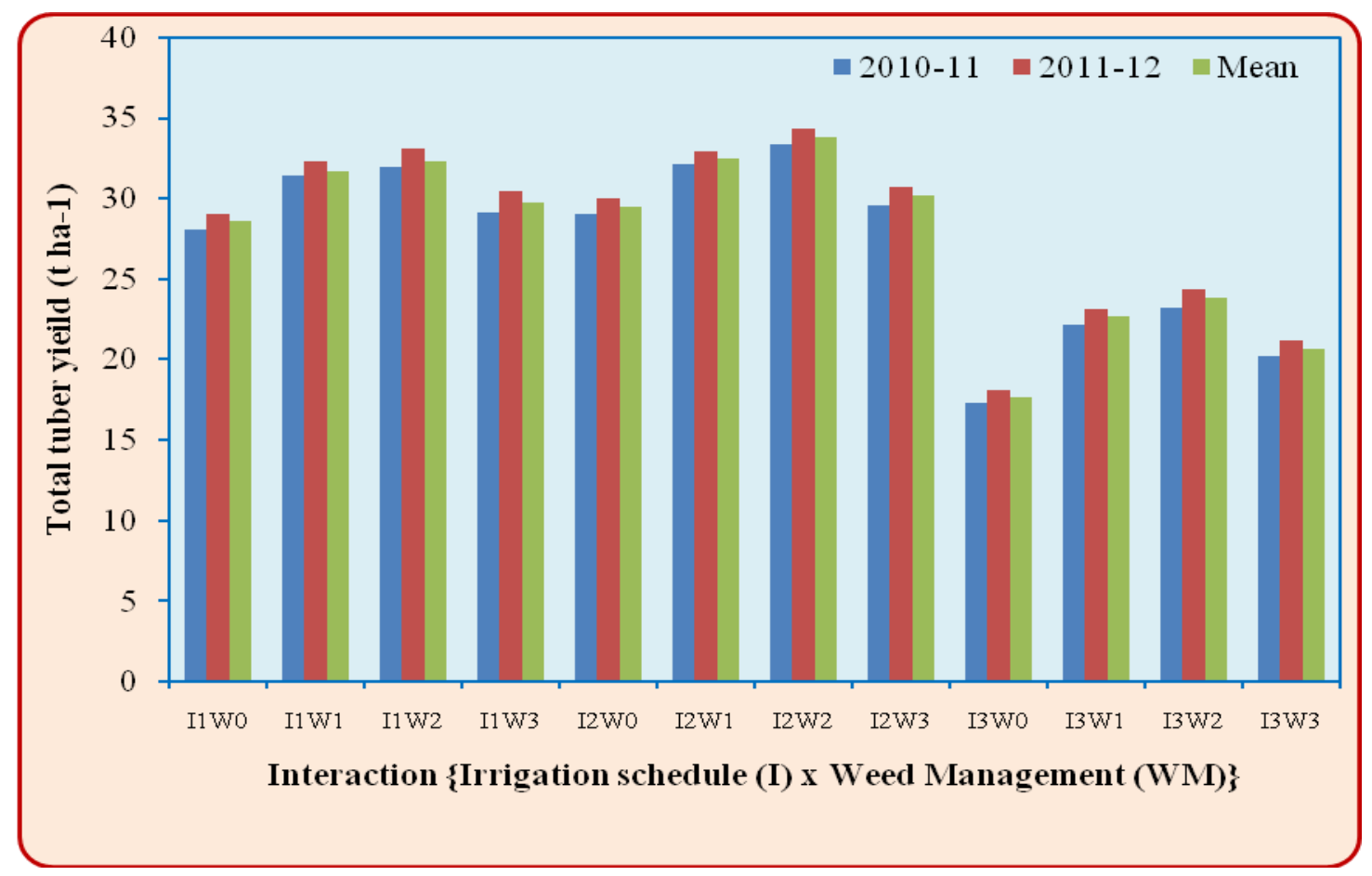


Fig.2 Effect of interaction between weed management (W) and integrated nutrient management $(\mathrm{F})$ on total tuber yield of potato $(\mathrm{t}$ ha- 1 )

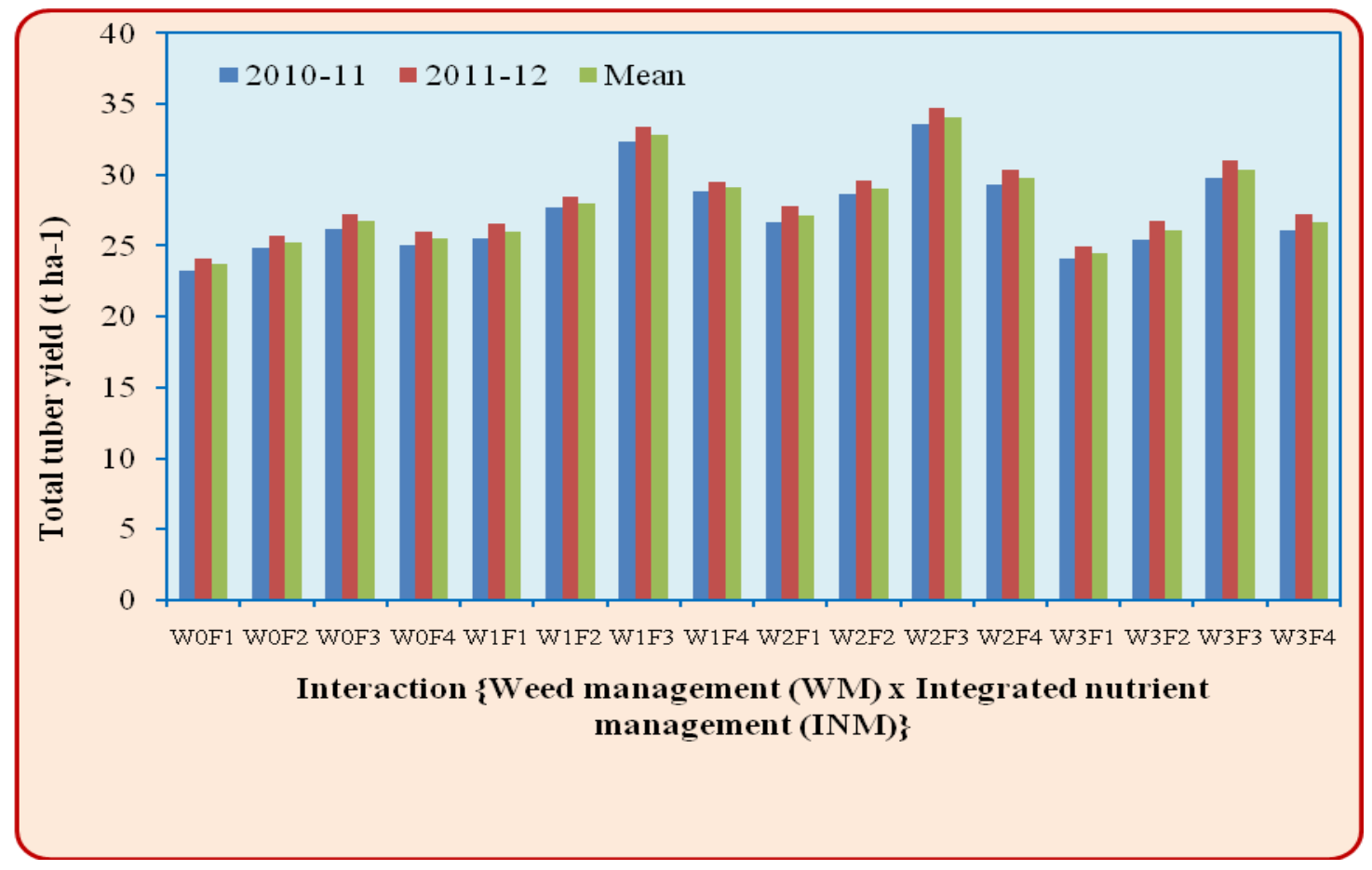

Fig.3 Effect of interaction between irrigation schedule (I) and integrated nutrient management $(\mathrm{F})$ on total tuber yield of potato $(\mathrm{t}$ ha-1)

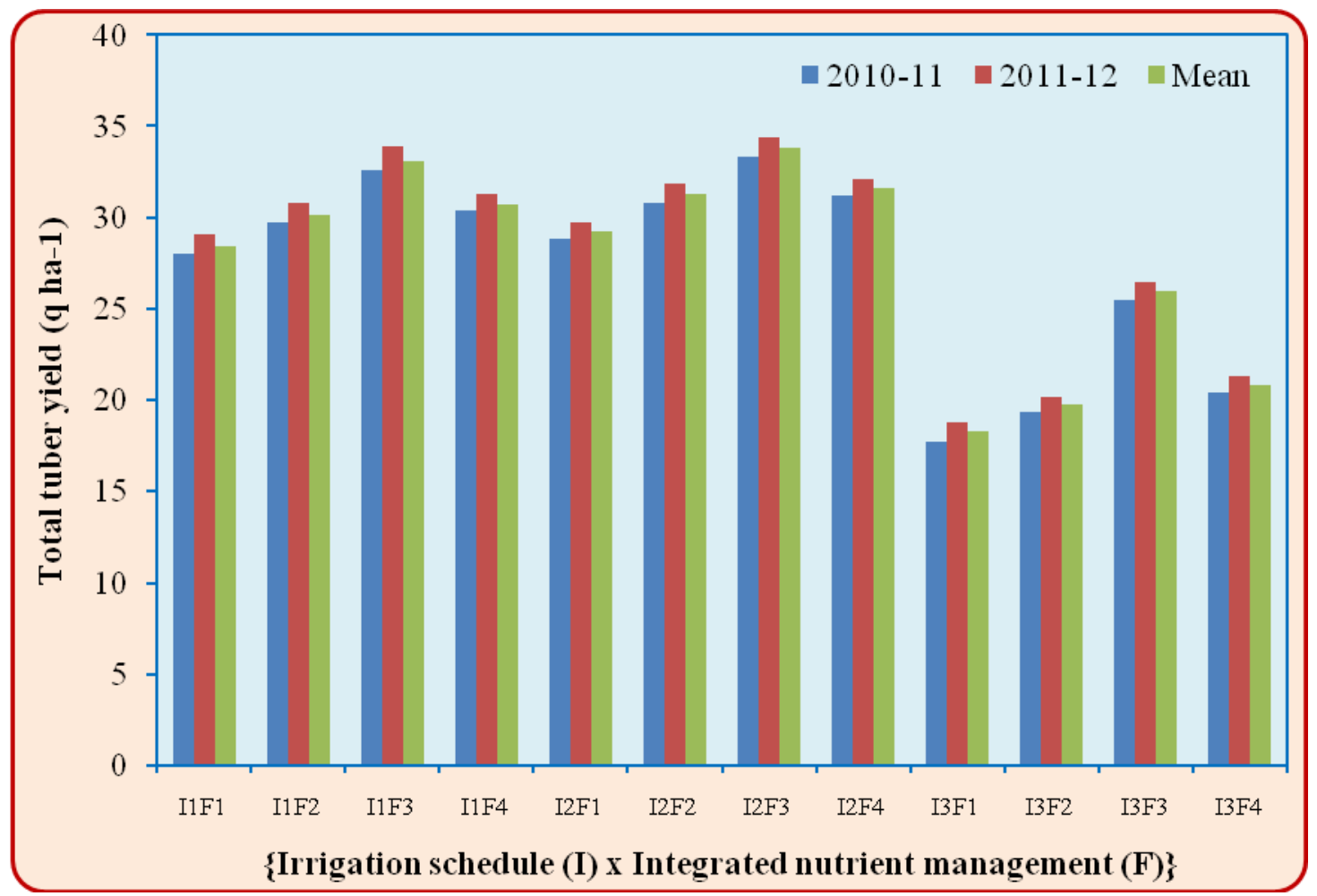


The higher yield attributing characters and yield was noticed in the above treatment which might be due to availability of water in sufficient quantity.

This may be due to the fact that water was applied frequently in optimum amount through drip irrigation compared to uneven distribution through furrow irrigation. Higher moisture content enhanced the plant growth which enhance the photosynthetic rate, enhance dry weight of tuber and finally increased the tuber yield. Badra et al., (2012). Among weed management practices, the number of stolons/plant (28.85), number of tubers/plants (14.62) and tuber yield (29.99 t/ha), were significantly higher under Metribuzin (500 $\mathrm{g}$ a.i. ha ${ }^{-1} \mathrm{PE}$ ) than weedy check and rest of the treatments. Significantly higher number of stolons/plant (29.11), number of tubers/plants (15.05) and tuber yield (30.96 t/ha) was found under treatment $75 \% \mathrm{~N}$ inorganic fertilizer $+25 \% \mathrm{~N}$ organic (Poultry manure) + PSB + Azotobactor than other nutrient management practices during both the years and on mean basis. Tubers were good quality and size this was due to crop has got the all major and micro nutrients by organic and inorganic fertilizers, weeds were also control timely by herbicides and crop get sufficient moisture due to drip irrigation round the season so nutrients uptake by crops sufficiently which increase the tuber yield (Table 5).

The interaction between irrigation schedule with weed management, weed management with integrated nutrient management and irrigation schedule with integrated nutrient management significantly influenced the total tuber yield during both the years and in mean data.

The results revealed that interaction of drip irrigation (125\% of Open pan evaporation) with application of Metribuzin (500g a.i ha ${ }^{-1}$
P.E) produced significantly higher total tuber yield as compared to other interactions (Fig. 1). Interaction between Metribuzin (500g a.i $\mathrm{ha}^{-1}$ P.E) and $75 \% \mathrm{~N}$ inorganic fertilizer +25 $\% \mathrm{~N}$ organic (Poultry manure) + PSB + Azotobactor produced significantly higher total tuber yield as compared to other interactions, but it was at par to interaction of two hand weeding (25 and 45 DAP) with75\% $\mathrm{N}$ inorganic fertilizer $+25 \% \mathrm{~N}$ organic (Poultry manure) + PSB + Azotobactor (Fig. 2). Similarly, interaction of drip irrigation (125\% of Open pan evaporation) with $75 \% \mathrm{~N}$ inorganic fertilizer $+25 \% \mathrm{~N}$ organic (Poultry manure) + PSB + Azotobactor produced significantly higher total tuber yield as compared to other interactions but it was statistically similar to interaction between drip irrigation (100\% of open pan evaporation) and $75 \% \mathrm{~N}$ inorganic fertilizer $+25 \% \mathrm{~N}$ organic (Poultry manure) + PSB + Azotobactor (Fig. 3).

These findings are in agreement with those reported earlier by Arora et al., (2009), Bakeer et al., (2009) and Baishya et al., (2010).

\section{Economics}

Economics of potato was as influenced by irrigation schedule, weed management and integrated nutrient management (Table 6). The gross income, net income and benefit: cost ratio ( $\mathrm{B}: \mathrm{C}$ ratio) were maximum under drip irrigation (125\% open pan evaporation) followed by drip irrigation (100\% open pan evaporation). The lowest cost of cultivation, gross income, net income and benefit: cost ratio was recorded under furrow irrigation during both the years and in mean data. Among the weed management practices, Metribuzin (500 g a.i. ha $\left.{ }^{-1} \mathrm{PE}\right)$ registered higher gross income, net income and $\mathrm{B}: \mathrm{C}$ ratio as compared to other treatments. However, total cost of cultivation was higher 
in two hand weeding (25 and 45 DAP) followed by Metribuzin (500 g a.i. ha $\left.{ }^{-1} \mathrm{PE}\right)$ and Chlorimuron + Quizalofop $(6+50 \mathrm{~g}$ a.i $\left.\mathrm{ha}^{-1}\right)$. The lowest cost of cultivation was recorded in weedy check during both the years as well as in mean data. Among integrated nutrient management practices, application of $75 \% \mathrm{~N}$ inorganic fertilizer +25 $\% \mathrm{~N}$ organic (Poultry manure) $+\mathrm{PSB}+$ Azotobactor registered higher gross income, net income and $\mathrm{B}$ : $\mathrm{C}$ ratio than other treatments. The lowest gross income, net income and B:C ratio was noted under $100 \%$ RDF. However, higher cost of cultivation was noted under $100 \%$ RDF + Micro nutrient (Zinc Sulphate $25 \mathrm{kgha}^{-1}$ ) followed by $75 \% \mathrm{~N}$ inorganic fertilizer $+25 \% \mathrm{~N}$ organic (Poultry manure) $+\mathrm{PSB}+$ Azotobactor and $50 \% \mathrm{~N}$ inorganic fertilizer $+50 \% \mathrm{~N}$ organic (Poultry manure) + PSB + Azotobactor. The lowest cost of cultivation was obtained under $100 \% \%$ RDF during both the years and on mean basis. Similar findings were also reported by Nowacki et al., (2009), Roder et al., (2009) and Verma et al., (2011).

\section{References}

Ahmed, I. M., Shaheenuzzamn, M., Nadira, U. A., Ahmed, M. H. and Hossain, A. 2011. Performance of herbicide hammer $24 \mathrm{Ec}$ in potato field. Journal of Experimetnal Bioscience 2(1): $11-14$.

Arora, A., Tomar, S.S. and Gole, M.K. (2009). Yield and quality of potato as influenced by weed management practices and their residual study in soil. Agricult Sci Dig., 29 (2) 1-3.

Bakeer, G.A.A., El-Ebabi, F.G., El-Saidi, M.T. and Abdelghany A. R. E. (2009). Effect of pulse drip irrigation on yield and water use efficiency of potato crop under organic agriculture in sandy soils. Journal of Agriculture Engineering 26(2): 736- 765.

Badra, M.A., El-Tohamyb, W.A. and
Zaghloulc, A.M. 2012. Yield and water use efficiency of potato grown under different irrigation and nitrogen levels in an arid region. Agricultural Water Management 110: 9-15.

Baishya, L.K., Kumar, M. and Ghose, D.C. (2010). Effect of different proportion of organic and inorganic nutrients on productivity and profitability of potato (Solanum tuberosum) varieties in Meghalaya hills. Indian Journal of Agronomy 55(3): 230-234.

Chandrakar C.K., Shrivastava, G.K., Dwivedi, S.K., Sharma, D. and Saxena, R. (2012). Agro-techniques for increasing yield potential of potato under drip irrigation for Chhattisgarh plains. Ph.D Thesis, Agronomy, IGKV, Raipur, Chhattisgarh

El Saidi, M.T., Kassab, O.M., Okasha, E.M. and Abdelghany, A.R.E. (2010). Effect of Drip irrigation systems, water regimes and irrigation frequency on growth and quality of potato under organic agriculture in sandy soils. Australian Journal of Basic and Applied Sciences, 4(9): 4131-4141.

Jan, H., Muhammad, A. and Ali, A. (2004). Studies on weed control in potato in Pakhal Plains of Mansehra. Pakisthan Journal of Weed Science Research 10(3-4): 157-160.

Nowacki, W. 2009. Irrigation as a modify factor of profitability in potato production in ecological system. Journal of Research and Applications in Agricultural Engineering 54(4): 32-35

Parmar, D.K., Sharma, Akhilesh; Chaddha, Sanjay;Sharma, V.,, Vermani, A., Mishra, A., Gautam, G. and Kumar, V. 2007. Increasing potato productivity and profitability throough integrated plant nuterint system in the North Western Himalayas. Potato J. 34 (3-4): 209-215

Roder, W., Dochen T., Nidup, K. and Dorji, 
S. (2009). Weed management challenges in small-holder potato systems in Bhutan. Weed Research 49 (3): 300-307.

Sarkar, A., Sarkar, S. and Zaman, A. 2011. Growth and yield of potato as influenced by combination of organic manures and inorganic fertilizers. Potato Journal 38 (1): 78-80.

Singh, V. P., Mishra, J.S. and Yaduraju, N. T. (2002). Impact of irrigation levels and metribuzin on weed growth and tuber yield of potato (Solanum tuberosum) under Vertisols. Indian Journal of Agricultural Sciences 72(3): 174-176.

Singh, S.N., Singh, B.P., Singh, O.P., Singh,
R. and Singh, R.K. 2007. Effect of nitrogen application in conjunction with bio-inoculants on the growth, yield and quality of potato under Indo-Gangetic Plain Region. Potato Journal 34 (1-2): 103-104.

Swalin,G., Raghav,M. 2008. Effect of integrated nutrient management on yield and quality of potato (Solanum tuberosum L.). Pantnagar Journal of Research. 6(1): 122-124

Verma, S.K., Asati, B.S, Tamrakar, S.K., Nanda, H.C. and Gupta, C.R. 2011. Effect of organic components on growth, yield and economic returns in potato. Potato Journal 38 (1): 51-55

\section{How to cite this article:}

Chandrakar, C.K., G.K. Shrivastava, S.K. Dwivedi, D. Sharma, K.K. Pandey and Singh, S.K. 2017. Advance Agro- Techniques for Yield Efficiency and Economics of Potato (Solanum tuberosum) Crop for Chhattisgarh Plains. Int.J.Curr.Microbiol.App.Sci. 6(2): 766-777. doi: http://dx.doi.org/10.20546/ijcmas.2017.602.085 